\title{
Membranas Compuestas: propiedades superficiales y estabilidad térmica
}

\author{
Composite membranes: surface properties \\ and thermal stability
}

Betina Villagra Di Carlo ${ }^{1}$, Alberto Claudio Habert ${ }^{2}$

\author{
${ }^{1}$ INIQUI-CONICET, CIUNSA, Universidad Nacional de Salta, Av. Bolivia 5150, CP:4400, Salta, Argentina \\ e-mail: betinadicarlo@gmail.com.ar \\ ${ }^{2}$ PEQ-COPPE, Universidad Federal de Río de Janeiro, Río de Janeiro, Brasil \\ e-mail: habert@ coppe.ufrj.br
}

\begin{abstract}
RESUMEN
Las membranas compuestas fueron preparadas con poli(alcohol vinílico) entrecruzado sobre membranas porosas de polietersulfona por el proceso de recubrimiento. Las membranas de PES fueron expuestas al plasma de aire con el propósito de modificar las propiedades superficiales. La capa de recubrimiento se preparó usando una matriz polimérica con un agente entrecruzamiento. Las propiedades físico-químicas se han estudiado por espectroscopía de infrarrojo con reflectancia total atenuada horizontal (FTIR / HATR) y medidas de ángulo de contacto. La estabilidad térmica y propiedades de transición de fases fueron investigadas por análisis térmico gravimétrico (TGA) y calorimetría diferencial de barrido (DSC). El poli(alcohol vinílico) es un polímero soluble en agua debe ser entrecruzado por reacciones de modificación con un agente de entrecruzamiento para formar una membrana estable con buenas propiedades mecánicas en pervaporación. Se utilizó PVA como polímero denso con ácido maléico como agente de entrecruzamiento. Los espectros de infrarrojo revelaron la naturaleza química de la superficie. Las medidas de ángulo de contacto evaluaron los cambios en las propiedades hidrofílicas. El tratamiento con plasma de aire causó una eficaz modificación de la superficie del soporte. El mecanismo responsable por la mejora en la mojabilidad fue la funcionalización superficial por inserción de grupos polares. La introducción de ácido maléico en la cadena del polímero aumentó la región amorfa de la membrana. El PVA es un polímero semi-cristalino que exhibe ambos una temperatura de transición vítrea $(\mathrm{Tg})$ y una isoterma de fusión $(\mathrm{Tm})$, como fue evidenciado en los termogramas de DSC. Se mejoraron significativamente las propiedades térmicas de las membranas compuestas, ambas, la temperatura de descomposición y el peso residual aumentaron.
\end{abstract}

Palabras clave: Membranas compuestas, polímeros, síntesis, caracterización, hidrofilia.

\section{ABSTRACT}

The composite membranes were manufactured with crosslinking poly(vinyl alcohol) on polyethersulfone porous membranes by coating process. PES membranes were exposed to air plasma for the purpose of surface modification. The coating of the support membrane was carried out by using a polymer matrix with a crosslinking agent. The physico-chemical properties have been studied by attenuated total reflectance fourier transform infrared spectroscopy (ATR-FTIR) and contact angle measurements. Thermal stability properties and phases transition of the composite membranes were investigated by thermal gravimetric analysis (TGA) and differential scanning calorimetry (DSC). Poly(vinyl alcohol) is water-soluble, hydrophilic and biodegradable polymer with excellent film forming properties. However, PVA must be crosslinked by modification reactions with a crosslinking agent to form a stable membrane with good mechanical properties in pervaporation. PVA was used as the dense polymer with maleic acid as a crosslinking agent. Fourier transformed infrared spectroscopy revealed the nature of the surface chemical. Contact angle measurements have been used for evaluating changes in wetting properties. The air-plasma treatment caused an effective surface modification. The mechanism responsible for the wettability improvement was the surface 
functionalization by insertion of polar groups. Introduction of maleic acid into the polymer chain increased the amorphous region of the membrane. The PVA is a semi-crystalline polymer exhibiting both a glass transition temperature ( $\mathrm{Tg}$ ) and a melting isotherm (Tm), as evidenced in the DSC thermogram. Thermal properties of the composite membranes were significantly enhanced. Both, the decomposition temperature and the residual weight increased when compared with dense PVA membranes. The chemically crosslinked PVA with maleic acid resulted in the formation of composite membranes with improved pervaporation properties for separation application.

Keywords: Composite membranes, polymers, synthesis, characterization, hydrophilicity.

\section{INTRODUCCIÓN}

Los procesos de separación que utilizan membranas poliméricas son investigados en aplicaciones industriales que incluyen las petroquímicas, bio-combustibles y aplicaciones ambientales de recuperación de compuestos químicos y tratamiento de aguas residuales. Las ventajas de estos procesos por membranas sobre los procesos convencionales de separación son; bajo consumo energético, no necesitan el agregado de aditivos para producir la separación, pueden ser operados en forma continua, son simples desde el punto de vista operacional, siendo además una tecnología ambientalmente limpia [1,2].

En pervaporación se utilizan membranas densas o compuestas debido al mecanismo involucrado en la separación (solución-difusión). Las membranas compuestas utilizan una membrana microporosa como soporte mecánico para una fina piel de un material polimérico denso depositado [3,4]. El soporte poroso aporta resistencia mecánica a la membrana con mínima resistencia al transporte (del orden de membranas porosas de ultrafiltración). También debe prestarse atención a la integridad estructural de la membrana compuesta sometida a las condicones de operación (temperatura y composición de la alimentación). La membrana polimérica actúa como barrera separativa para el proceso de separación de pervaporación. La estructura de la membrana compuesta define el mecanismo de transporte de las diferentes especies permeantes.

La membrana porosa substrato puede ser sintetizada por el proceso de inversión de fase, combinando la técnica en seco de evaporación del solvente con la técnica de precipitación por inmersión. La capa densa selectiva puede depositarse por la técnica de recubrimiento con solución polimérica y evaporación controlada del solvente (casting) [5,6]. Al cubrir la superficie de las membranas asimétricas soportes con una fina membrana densa se obtienen membranas anisotrópicas compuestas. La piel densa depositada cubre los defectos superficiales de las membranas soportes y modifica las propiedades de transporte de masa a través de la membrana $[7,8]$.

El PVA [poli(alcohol vinílico)] es un polímero semicristalino, constituido por una fase amorfa y una cristalina. Posee características únicas como formador de películas, es utilizado en diversas aplicaciones como adhesivo, engomante y espesante, debido a su solubilidad en agua. También es un polímero de baja toxicidad y biocompatible bajo investigación en aplicaciones biomédicas [9]. Para lograr una estabilidad química adecuada, una membrana constituida de PVA necesita ser entrecruzada antes de aplicarse a separaciones de mezclas por pervaporación [10]. El agregado de un agente reticulante (acido maleico) seguido de tratamiento térmico es adecuado para este propósito.

Los tratamientos con plasmas permiten modificar las propiedades físico-químicas superficiales de membranas poliméricas de forma efectiva y menos contaminante que otros procesos convencionales (recubrimientos, injertos, aditivos) [8,11]. Las principales variables que determinan la modificación por plasma son: la composición química de la membrana soporte, la naturaleza del gas o vapor alimentado, el tiempo de modificación, la presión en la cámara de reacción, la potencia de la descarga de radio-frecuencia basado en $[12,13]$.

En este trabajo fueron sintetizadas membranas planas porosas asimétricas con morfología adecuada para la deposición de una fina membrana densa por la técnica de casting. Se evaluaron propiedades superficiales y térmicas de las membranas compuestas de PVA/PES (polietersulfona).

\section{MATERIALES Y MÉTODOS}

\subsection{Materiales}

La síntesis de las membranas porosas fue realizada con PES (P.M. $\approx 58.000 \mathrm{~g} / \mathrm{mol}$, Basf). El aditivo soluble formador de poros fue polivinilpirrolidona (PVP, P.M. $\approx 40.000 \mathrm{~g} / \mathrm{mol}$, Sigma) y el solvente fue dimetilformamida (DMF, Merck). Se utilizo agua destilada para el baño de no-solvente en la precipitación de 
la membrana porosa. El polímero seleccionado para depositar la capa densa fue PVA (P.M. $\approx 72.000 \mathrm{~g} / \mathrm{mol}$, $80 \%$ hidrolizado, Vetec]. El agente reticulante incorporado a la solución de recubrimiento fue acido maleico (Vetec).

\subsection{Membranas porosas asimétricas soporte}

Se utilizó la técnica de inversión de fase por inmersión para sintetizar las membranas porosas soportes. La solución polimérica (15\% PES, 7.5\% PVP y 77.5\% DFM en peso) fue esparcida sobre placas de vidrio rectangulares. El tiempo de evaporación parcial del solvente fue 20 segundos. El baño de no-solvente para la precipitación de la membrana fue agua destilada a temperatura ambiente. Las membranas fueron lavadas con agua destilada, luego con etanol y por último con n-hexano.

\subsection{Tratamiento superficial por plasma}

La activación del soporte polimérico fue realizada por la técnica de tratamiento por plasma de gas nopolimerizable (aire). Un reactor inductivo de radio frecuencia (Harrick, $8-12 \mathrm{MHz}$ ) fue utilizado en la modificación por plasma. El aire fue alimentado a temperatura ambiente. La potencia de la descarga fue fijada en $29.6 \mathrm{~W}$ y la presión de la cámara de reacción en $650 \mathrm{mTorr}$. El soporte poroso fue tratado con el plasma de aire durante 10 minutos.

\subsection{Membranas compuestas}

El recubrimiento con PVA fue realizado por la técnica de solución-evaporación (casting). La solución polimérica (5\% $/ \mathrm{p}$. de PVA) con agente reticulante (acido maleico, $0.5 \% \mathrm{p} / \mathrm{p}$.) fue esparcida sobre las membranas porosas. El solvente (agua destilada) fue evaporado lentamente a temperatura ambiente. Las membranas compuestas secas fueron sometidas a tratamiento térmico $\left(150^{\circ} \mathrm{C}, 3\right.$ horas) con el fin de promover reacciones de reticulación.

\subsection{Caracterizaciones}

La composición química superficial fue analizada por espectroscopía de infrarrojo con reflectancia total atenuada horizontal (FTIR-HATR, Perkin-Elmer, Spectrum GX), los espectros fueron barridos 12 veces, entre 4000 y $630 \mathrm{~cm}^{-1}$, con resolución de $4 \mathrm{~cm}^{-1}$. Las propiedades hidrofílicas superficiales fueron investigadas midiendo el ángulo de contacto $(\theta)$ con agua destilada utilizando un goniómetro (Ramé-Hart, con DROPimage), el software del equipamiento estima la energía superficial $(\gamma \mathrm{S})[4,8]$. Las transiciones térmicas (temperatura de transición vítrea [Tg] y de fusión [Tm]) de las membranas de PVA $80 \%$ hidrolizado reticulado con ácido maleico y tratamiento térmico $\left(150^{\circ} \mathrm{C}, 3\right.$ horas) fueron determinadas por DSC (PerkinElmer, modelo DSC-7), el rango de temperatura fue entre 0 y $250{ }^{\circ} \mathrm{C}$ con una velocidad de calentamiento de $10{ }^{\circ} \mathrm{C} / \mathrm{min}$ bajo atmósfera de gas inerte (nitrógeno). Las etapas de degradación térmica (cantidad de etapas, temperatura de degradación) de las membranas de PVA $80 \%$ hidrolizado con ácido maleico, sin tratamiento térmico y las reticuladas con ácido maleico y tratamiento térmico $\left(150^{\circ} \mathrm{C}, 3\right.$ horas $)$ fueron evaluadas por TGA (Perkin-Elmer, modelo TGA 7), el rango de temperatura fue entre 50 y $700{ }^{\circ} \mathrm{C}$ a una velocidad de $10^{\circ} \mathrm{C} /$ min en atmósfera de gas inerte (nitrógeno).

\section{RESULTADOS Y DISCUSIÓN}

\subsection{Naturaleza química}

El PVA es soluble en agua debido a la presencia de grupos hidrofílicos en su estructura. Por lo tanto es necesario modificar la estructura del PVA mediante reacciones de entrecruzamiento. Las membranas preparadas a partir de este polímero fueron sometidas a tratamientos térmicos y químicos, con el objetivo de promover ligazones cruzadas en la matriz polimérica hidrofílica. El tratamiento térmico fue efectuado a $150^{\circ} \mathrm{C}$ (temperatura entre la transición vítrea y la temperatura de fusión) durante 3 horas para completar las reacciones de reticulación. El agente reticulante utilizado fue el ácido maleico. El entrecruzamiento proporciona resistencia química, las propiedades térmicas de las membranas de PES sintetizadas como soporte poroso, permiten el tratamiento térmico de la membrana densa depositada.

La Figura 1 presenta el espectro de IR-FT-HATR para la membrana compuesta PVA/PES después del tratamiento térmico. Se pueden observar las bandas correspondientes a la vibración del estiramiento de los grupos O-H $\left(3300 \mathrm{~cm}^{-1}\right)$, las vibraciones del estiramiento asimétrico del C-H $\left(2936 \mathrm{~cm}^{-1}\right)$ y simétrico $(2911$ $\left.\mathrm{cm}^{-1}\right)$, las vibraciones de la deformación del C-H2 $\left(1420 \mathrm{~cm}^{-1}\right)$ y $\left(1328 \mathrm{~cm}^{-1}\right)$, las del estiramiento del C-O en 
$\left(1241 \mathrm{~cm}^{-1}\right)$ y $\left(1090 \mathrm{~cm}^{-1}\right)$ y del doblamiento del O-H a $\left(917 \mathrm{~cm}^{-1}\right)$ según lo encontrado por [14,15].

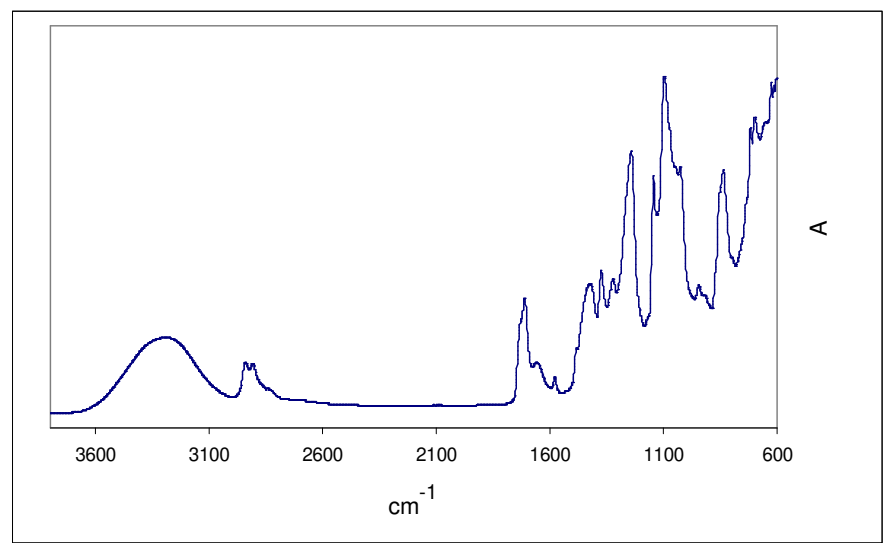

Figura 1: Espectro FT-IR/HATR de una membrana compuesta de PVA 80\% hidrolizado reticulado con ácido maleico y tratamiento térmico.

El PVA al ser parcialmente hidrolizado posee grupos acetatos en la cadena polimérica que corresponden a la banda de absorción localizada alrededor de $1730 \mathrm{~cm}^{-1}$ relativa a los grupos carbonilos. Se observa un pico alrededor de los 1250-1260 $\mathrm{cm}^{-1}$ en las membranas entrecruzadas, lo cual indica la formación de uniones éter (-C-O-C-) entre las cadenas de PVA con el agente reticulante (Fig. 1), [10,16]. La reticulación de las cadenas poliméricas por tratamiento químico y térmico evitó la disolución de la capa selectiva de la membrana compuesta durante los ensayos de pervaporación con mezclas acuosas [6].

Los espectros de infrarrojo de la superficie formada muestran la aparición de bandas de absorción correspondientes al PVA sobre la membrana de PES, indicando la formación de la membrana compuesta (Fig. 1).

\subsection{Propiedades hidrofílicas superficiales}

Las superficies de las membranas fueron estudiadas usando medidas de ángulo de contacto debido a la facilidad y rapidez del método en examinar propiedades superficiales, proporciona información de la hidrofília de las membranas $[8,11,17]$.

El tratamiento con plasma de aire $(29.6 \mathrm{~W}, 650 \mathrm{mTorr}, 10 \mathrm{~min})$ modificó el grado de mojado de la superficie de las membranas porosas de PES. Las membranas soporte de PES exhibieron una importante disminución del ángulo de contacto, aumento de la energía superficial y del trabajo de adhesión después del tratamiento con plasma generado a partir de aire. Las membranas compuestas son hidrofílicas y estables, la medida del ángulo de contacto de las películas depositadas indica un valor de $53^{\circ} \pm 1$.

Antes del proceso de recubrimiento con PVA, la membrana soporte fue sometida a tratamiento por plasma de aire, la función de esta etapa fue funcionalizar la superficie modificándola hidrofílicamente. La superficie de la membranas de PES sin tratamiento posee características hidrofóbicas con un ángulo de contacto de $66^{\circ}$ y una energía superficial de $42.32 \mathrm{~mJ} / \mathrm{m}^{2}$. La funcionalización del soporte con el tratamiento por plasma de aire disminuyó el ángulo de contacto a $24^{\circ}$. La etapa de recubrimiento con PVA depositó una piel de polímero denso con ángulo de contacto de $\theta=53^{\circ}$ y $\gamma \mathrm{S}=51.68 \mathrm{~mJ} / \mathrm{m}^{2}$ de energía superficial. El carácter hidrofílico de la membrana permite la permeación de agua con flujos y selectividades que dependen de la composición química de la capa activa y morfología de la membrana $[6,18]$.

\subsection{Transiciones térmicas}

La curva del flujo endotérmico de calor en función de la temperatura para una membrana densa de PVA 80\% hidrolizado, reticulada con ácido maleico y tratamiento térmico, se presenta en la figura (2). La temperatura de transición vítrea muestra un valor de $69.4{ }^{\circ} \mathrm{C}$, mientras que la temperatura de fusión corresponde a $175^{\circ} \mathrm{C}$. Se puede observar la presencia de regiones cristalinas en el PVA después de la reticulación $(\Delta \mathrm{H}=33.6 \mathrm{~J} / \mathrm{g})$, lo que corresponde a un porcentaje de cristalinidad de $20.1 \%$ (Ec. 1). 
cristalinidad $\quad(\%)=\left(\frac{\Delta H_{f s}}{\Delta H_{f r}}\right) \cdot 100$

donde $\Delta H_{f s}$ es el cambin en el calor de fusión de la muestra, el cual puede obtenerse directamente de los gráficos de DSC (Fig 2). $\Delta H_{f r}$ es el cambio en el calor de fusión para el PVA de referencia (cristalinidad 100\%) y normalmente se toma como $167 \mathrm{~J} / \mathrm{g}$ [19].

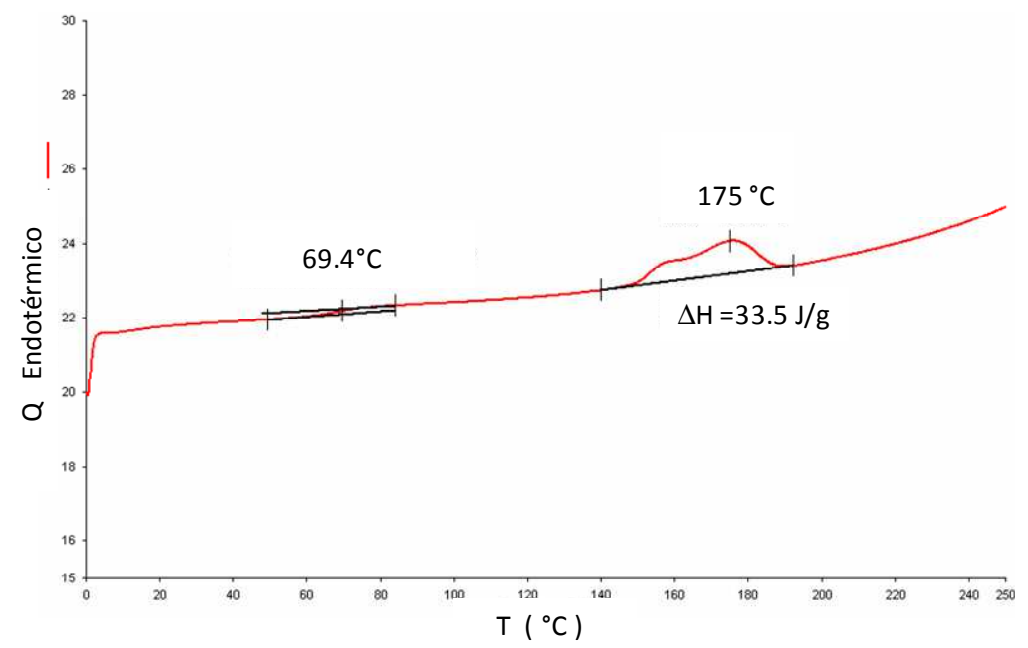

Figura 2: Curvas del análisis por DSC de una membrana densa de PVA 80\% hidrolizado, reticulada con ácido maleico y tratamiento térmico.

Las membranas de PVA entrecruzadas muestran un decrecimiento en la temperatura de fusión después del entrecruzamiento con acido maléico, sugiriendo un arreglo menos ordenado de las cadenas de PVA luego de introducir el acido maléico y someterlo al tratamiento térmico de reticulación. La introducción del agente reticulante (ácido maleico) afecta el porcentaje de fase cristalina y los espacios entre los segmentos de la cadena polimérica en la región amorfa, lo cual modifica la temperatura de transición vítrea. Las reacciones de reticulación consumen grupos hidroxilos, lo que conduce a una disminución de la rigidez comparando con el polímero puro. Por otro lado, el entrecruzamiento reduce la movilidad segmental de las cadenas poliméricas lo que provoca aumento de la rigidez [9].

La membrana reticulada presenta $\mathrm{Tg}$ superior al polímero puro y disminución del porcentaje de fase cristalina debido a que la efectiva reacción de entrecruzamiento aumenta la rigidez del polímero, como también se comprobó en los experimentos de sorción [20].

\subsection{Estabilidad térmica}

La curva de decrecimiento de la masa como una función de la temperatura de una membrana densa de PVA $80 \%$ hidrolizado con ácido maleico, sin tratamiento térmico, se presenta en la figura (3). El PVA con ácido maleico se degrada en tres etapas. La primera ocurre antes de $\operatorname{los} 200^{\circ} \mathrm{C}$, la segunda entre $\operatorname{los} 280^{\circ} \mathrm{C}$ y $\operatorname{los}$ $400^{\circ} \mathrm{C}$, y la tercera entre $\operatorname{los} 400^{\circ} \mathrm{C}$ y $\operatorname{los} 477^{\circ} \mathrm{C}$. La primera etapa de degradación probablemente se debe a la evaporación de agua dado que es un polímero hidrofílico y a la descomposición térmica del ácido maléico agregado. La segunda etapa incluye la descomposición de las cadenas por deshidratación del polímero acompañada por la formación de productos volátiles y polímeros insaturados (residuos). En la tercera etapa, el residuo polimérico es degradado [20]. 


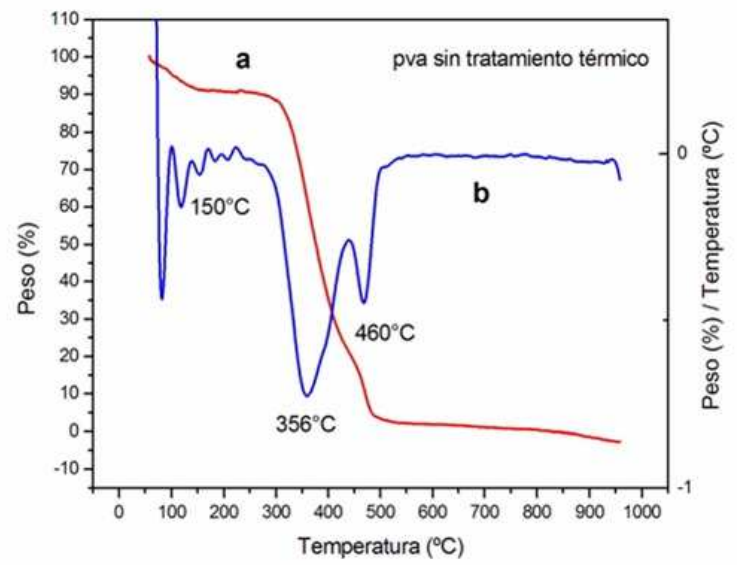

Figura 3: Curvas del análisis termogravimétrico de una membrana densa de PVA $80 \%$ hidrolizado con ácido maleico, sin tratamiento térmico, a) termograma por TGA, b) derivada del termograma.

Las curvas de termogravimetría para las membranas compuestas reticuladas es presentada en la figura (4), donde puede observase que el polímero reticulado presenta dos etapas de degradación. Se observa del termograma que el perfil de degradación fue alterado, por tratarse de una membrana compuesta y por el tratamiento térmico que promovió uniones entrecruzadas, cambiando el mecanismo de ruptura de cadenas (Fig. 4). Estos resultados confirman los resultados obtenidos con anterioridad por sorción [20], que indicaron la efectividad de la reticulación a una temperatura de $150^{\circ} \mathrm{C}$ durante 3 horas.

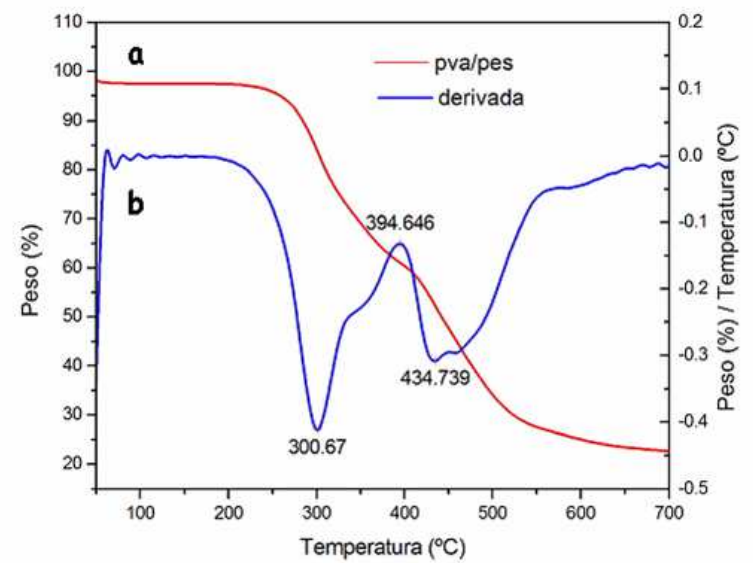

Figura 4: Curvas del análisis termogravimétrico de una membrana compuesta de PVA $80 \%$ hidrolizado reticulada con ácido maleico y tratamiento térmico, a) termograma por TGA, b) derivada del termograma.

Además en las membranas reticuladas ocurrió un aumento de la estabilidad térmica, como es evidenciado por el aumento de la fracción de masa residual (60\% del peso inicial) en que se inicia la segunda etapa de degradación de la membrana. Esto también se relaciona con la masa del substrato de PES que posee mayor resistencia térmica que las capas depositadas. Estas membranas compuestas reticuladas no presentan degradación debajo de $\operatorname{los} 200^{\circ} \mathrm{C}$ y fueron aplicadas a pervaporación donde la máxima temperatura de operación fue de $60^{\circ} \mathrm{C}$ [6]. Lo cual sugiere que la estabilidad térmica de la membrana de PVA aumento significativamente debido al soporte y al entrecruzamiento de la capa depositada. 


\section{CONCLUSIONES}

El recubrimiento con PVA formó una membrana compuesta sobre el soporte poroso de PES. El análisis de la composición química superficial por espectroscopía de infrarrojo de las membranas compuestas demuestra la fuerte presencia de bandas asignadas al $\mathrm{O}-\mathrm{H}\left(3300 \mathrm{~cm}^{-1}\right)$ y $\mathrm{C}=\mathrm{O}\left(1725 \mathrm{~cm}^{-1}\right)$ que revelan el carácter hidrofílico de las membranas depositadas. El tratamiento superficial por plasma de aire de la membrana porosa produjo una disminución en el ángulo de contacto $(\theta)$ lo que evidencia el aumento de hidrofilia y energía superficial $(\gamma \mathrm{S})$. Después de la reticulación se observa la presencia de regiones cristalinas en el PVA $(\Delta \mathrm{H}=33.6 \mathrm{~J} / \mathrm{g})$, que corresponde a un porcentaje de cristalinidad del $20.1 \%$. Las propiedades térmicas de las membranas de PES sintetizadas como soporte poroso, permiten el tratamiento térmico de la membrana compuesta preparada. En las membranas reticuladas ocurrió un incremento de la estabilidad térmica evidenciado por el aumento de la cantidad de masa residual en que se inicia la segunda etapa de degradación.

\section{AGRADECIMIENTOS}

Los autores agradecen a CONICET, CIUNSA, CAPES, COPPE.

\section{BIBLIOGRAFÍA}

[1] SMITHA, B., SUHANYA, D., SRIDHAR, S., et al., "Separation of organic-organic mixtures by pervaporation- review", Journal of Membrane Science, v. 241, pp. 1-21, 2004.

[2] HABERT, A., BORGES, C., NÓBREGA, R., Processos de Separação por Membranas, 1 ed., Rio de Janeiro, Coppe-Ufrj, 2006

[3] LEE, K., TENG, M., LEE, H., et al., "Dehydration of ethanol/water mixtures by pervaporation with composite membranes of polyacrylic acid and plasma-treated polycarbonate", Journal of Membrane Science, v. 164 , pp. 13-23, 2000.

[4] VILLAGRA DI CARLO, B., GOTTIFREDI, J., et al., "Pervaporation through composite membranes with plasma treatment of porous support ", Desalination and Water Treatment, v. 27, pp. 1-6, 2011.

[5] GUO, R., FANG, X., WU, H., et al., "Preparation and pervaporation performance of surface crosslinked PVA/PES composite membrane", Journal of Membrane Science, v. 322, pp. 32-38, 2008.

[6] VILLAGRA DI CARLO, B., HABERT, A., "Plasma-treated polyethersulfone coated with crosslinked poly(vinyl alcohol): composite membranes for pervaporation dehydration of ethanol", Journal Materials Science, v. 48, pp. 1457-1464, 2013.

[7] BAKER, R., Membrane Technology and Applications, 2 ed., John Wiley \& Sons, Ltd., 2004.

[8] VILLAGRA DI CARLO, B., GOTTIFREDI, J., HABERT, A., "Synthesis and characterization of composite membrane by deposition of acrylic acid plasma polymer onto pre-treated polyethersulfone support”, Journal Materials Science, v. 46, pp. 1850-1856, 2011.

[9] HASIMI, A., STAVROPOULOU, A., PAPADOKOSTAKI, K., et al., "Transport of water in polyvinyl alcohol films: Effect of thermal treatment and chemical crosslinking", European Polymer Journal, v. 44 pp. 4098-4107, 2008.

[10] GUO, R., HU, C., LI, B., et al., "Pervaporation separation of ethylene glycol/water mixtures through surface crosslinked PVA membranes: Coupling effect and separation performance analysis", Journal of Membrane Science, v. 289, pp. 191-198, 2007.

[11] KULL, K., STEEN, M., FISHER, E., "Surface modification with nitrogen-containing plasmas to produce hydrophilic low-fouling membranes", Journal of Membrane Science, v. 246 , pp.203-215, 2005.

[12] WAVHAL, D., FISHER, E., "Hydrophilic modification of polyethersulfone membranes by low temperature plasma-induced graft polymerization", Journal of Membrane Science, v. 209, pp. 255-269, 2002.

[13] FENG, J., WEN, G., HUANG, W., et al., "Influence of oxygen plasma treatment on poly(ether sulphone) films", Polymer Degradation and Stability, v. 91, pp. 12-20, 2006.

[14] WANG, L., LI, J., LIN, Y., et al., "Crosslinked poly(vinyl alcohol) membranes for separation of dimethyl carbonate/methanol mixtures by pervaporation", Chemical Engineering Journal, v.146, pp. 71-78, 2009.

[15] MAGALAD, T., GAVISIDDAPPA, S., GOKAVI, K., et al., "Mixed matrix blend membranes of poly(vinyl alcohol)-poly(vinyl pyrrolidone) loaded with phosphomolybdic acid used in pervaporation dehydration of ethanol", Journal of Membrane Science, v. 354, pp. 150-161, 2010. 
[16] KANG, M., CHOI, Y., MOON, S., "Water-swollen cation-exchange membranes prepared using poly(vinyl alcohol) (PVA)/poly(styrene sulfonic acid-co-maleic acid) (PSSA-MA)", Journal of Membrane Science, v. 207, pp. 157-170, 2002.

[17] VILlAGRA DI CARLO, B., CASTRO, E., HABERT, A., "Síntesis y caracterización de películas plasma hidrofílicas sobre membranas poliméricas anisotrópicas”, In: Proceedings of VIIth Ibero-American Conference on Membrane Science and Technology, pp. 262-263, Lisboa, Portugal, Marzo 2010.

[18] VILlAGRA DI CARLO, B., CASTRO, E., HABERT, A., "Recubrimientos hidrofílicos depositados sobre membranas porosas de polietersulfona", In: Proceedings of VIII Congreso Ibero-Americano en Ciencia y Tecnología de Membranas, pp. 90-91, Salta, Argentina, Mayo 2012.

[19] ZHANG, W., ZHANG, Z., WANG, X., "Investigation on surface molecular conformations and pervaporation performance of the poly(vinyl alcohol) (PVA) membrane", Journal of Colloid and Interface Science, v. 333, pp. 346-353, 2009.

[20] VILLAGRA DI CARLO, B., Desarrollo de membranas compuestas utilizando la tecnología de Plasma, Tesis de D.Sc., Universidad Nacional de Salta, Salta, 2010. 